\title{
Article
}

\section{Profile of repeat victimisation within multi-agency referrals}

Shorrock, Sarah, Mcmanus, Michelle Ann and Kirby, Stuart

Available at http://clok.uclan.ac.uk/31564/

Shorrock, Sarah, Mcmanus, Michelle Ann ORCID: 0000-0002-0095-1071 and Kirby, Stuart ORCID: 0000-0002-3049-1248 (2020) Profile of repeat victimisation within multi-agency referrals. International Review of Victimology, 26 (3). pp. 332-343. ISSN 0269-7580

It is advisable to refer to the publisher's version if you intend to cite from the work. http://dx.doi.org/10.1177/0269758020902890

For more information about UCLan's research in this area go to http://www.uclan.ac.uk/researchgroups/ and search for <name of research Group $>$.

For information about Research generally at UCLan please go to http://www.uclan.ac.uk/research/

All outputs in CLoK are protected by Intellectual Property Rights law, including Copyright law. Copyright, IPR and Moral Rights for the works on this site are retained by the individual authors and/or other copyright owners. Terms and conditions for use of this material are defined in the policies page.

\section{CLoK}

Central Lancashire online Knowledge www.clok.uclan.ac.uk

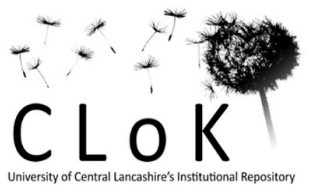



performance in court-sport athletes

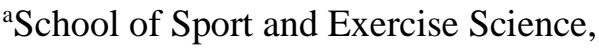
University of Lincoln,

\section{Acknowledgments}

18 Funding for this research was provided by the School of Sport and Exercise Science at the University of

19 Lincoln. There was no conflict of interest associated with the study. The authors would like to thank the 20 athletes who volunteered to participate in the research. 
Abstract

27 This study aimed to investigate the effects of an 8 week neuromuscular training protocol on dynamic 28 neuromuscular control and physical performance. Twenty female court-sport athletes were randomly 29 allocated to two groups, a control $(n=10)$ and an intervention group $(n=10)$. All participants performed 30 pre-testing inclusive of; a 20-m sprint, countermovement jump (CMJ), Illinois agility test and the qualitative 31 analysis of a single-leg squat (QASLS), which is an assessment of dynamic neuromuscular control. 32 Subsequently the intervention group engaged in a modified version of the FIFA 11+ neuromuscular training 33 programme twice weekly for eight weeks. At post-testing there were between-group differences in CMJ 34 (Intervention; +3.96-cm vs Control; -1.36-cm, p = 0.003) and QASLS for both legs $(\mathrm{p}<0.001)$ (Right-Leg, 35 Intervention; Pre: 3.75 Post: 1.91, Control; Pre: 4.11 Post: 4.08) (Left-Leg, Intervention; Pre: 3.72 Post: 0.98, Control; Pre: 4.05 Post: 4.23). There was a within-group improvement in 20-m sprint for the intervention group only post-training (Pre: 3.69-s Post: 3.60-s, $\mathrm{p}=0.043$ ), whilst no differences occurred in Illinois agility test for either group. A modified FIFA 11+ protocol can be considered an effective neuromuscular training programme for enhancing dynamic neuromuscular control and sport-specific physical performance in female court-sport athletes. 
53 Court-sport athletes, such as those involved in badminton, squash, netball, basketball and volleyball are

54 required to repetitively perform movements patterns such as jumping, landing and changing direction quickly whilst competing. ${ }^{1,2}$ Irrespective of sex, the movement patterns performed by court-sport athletes inherently present a risk of injury if performed with compromised technique and body mechanics. ${ }^{3}$ Repetitive quick changes in direction can cause anterior translation of the tibia relative to femur, which places a significant amount of force on the anterior cruciate ligament (ACL). ${ }^{4}$ This excessive force exerted on the ACL can cause ligament rupture, with females reported to be up to eight times more likely to suffer an ACL injury in comparison to males. ${ }^{5}$ Subsequently, ACL annual injury risk in female court athletes (up to $7.3 \%$ of surveyed population suffering a current ACL injury) are some of the highest reported when compared to variety of sporting populations. ${ }^{6}$ The speculated reasons why females are at an increased risk of injuring their ACL include hormonal and anatomical factors which affect the rigidity of the ACL. ${ }^{7}$

Abnormal dynamic alignment and neuromuscular-control of the lower limb during athletic tasks such as jumping, landing and changing direction has been associated with numerous lower limb pathologies including ACL injury. ${ }^{3}$ Kinematic analysis of the single-leg squat has been shown to predict and mirror movement dysfunction in a netball specific leap-landing task, and subsequently is recognized as an effective field-based screening method for identifying netball players who display biomechanical deficits during landing. ${ }^{1}$ In addition, mixed sport elite collegiate athletes displaying poor movement performance on a single-leg squat had significantly greater subsequent incidence of lower extremity injury, when compared

72 (QASLS) scoring system was developed to quantify neuromuscular control at various body regions during 73 a single-leg squat. This method has demonstrated high validity in relation to 3D motion capture (percentage 74 of agreement; $98 \%$, range $97 \%-100 \%){ }^{9}$

Females often demonstrate neuromuscular deficiencies in comparison to males in actions such as 76 eccentric muscular landing control and trunk to lower-limb alignment during landing and changing 77 direction. ${ }^{3}$ Electromyography (EMG) research has demonstrated females appear to have a lower ability to 
recruit the muscle groups needed to protect the integrity of the ACL, notably the hamstrings group and local trunk stabiliers such as the transversus abdominis and multifidus. ${ }^{3,10}$ However, it has been demonstrated, that if females engage in an effective neuromuscular training programme it can increase their ability to recruit such protective muscles groups. ${ }^{11}$ Neuromuscular training programmes include plyometric, balance, perturbation and strength exercises. These forms of training induce specific neural adaptations within the descending corticospinal tracts, motor units, neuromuscular junctions as well as improving the reflex potential of the muscle spindles. ${ }^{12,13}$ One neuromuscular training programme that has demonstrated significant promise for decreasing the risk of injury and improving performance variables is the FIFA $11+.{ }^{14,15}$ Soligard et al. ${ }^{15}$ reported significant reductions in minor and severe injury within female soccer players performing the FIFA 11+ protocol over an eight-month period compared to a control group. Subsequent authors have also reported the FIFA 11+ programme to have beneficial effects in reducing injuries. ${ }^{11,14,16}$ The FIFA $11+$ protocol has shown to improve physical performance variables such jump height, and sprint ability when implemented both acutely and longitudinally as a training intervention. ${ }^{17,18}$ The FIFA 11+ protocol has demonstrated to reduce injury risk and increase performance indicators in mostly soccer players. ${ }^{14,15}$ However there is noticeable lack of research on how the FIFA $11+$ protocol benefits those in other sporting contexts. Considering a female's increased risk of an ACL injury and the additional risk of injuring this ligament through participating in a court-sport, ${ }^{3}$ it seems appropriate to investigate the effects of the FIFA 11+ programme on physical performance and lower limb neuromuscular control, which has been emphasised as a predictive marker of ACL injury. ${ }^{1,8}$

The purpose of this study was to investigate the effects of an 8 week neuromuscular training protocol on dynamic neuromuscular control of the lower limb and physical performance in female courtsport athletes. Subsequently, it was hypothesised that the intervention group performing neuromuscular training would experience significant improvements in 20-m sprint, agility performance, countermovement jump height and neuromuscular control of the lower-limb as assessed via single-leg squat, when compared 102 to the control group. 


\section{Materials and Methods}

105 Participants

106 Twenty female court-sport athletes participated (stature: $169 \pm 7$-cm, mass: $61.3 \pm 8.3-\mathrm{kg}$, age: $22.3 \pm 2.0$ -

107 years). The participants came from a variety of different sporting backgrounds which included badminton,

108 squash, futsal, netball, basketball and volleyball. Participants were randomly assigned to either an 109 intervention group $(\mathrm{n}=10)$ or a control group $(\mathrm{n}=10)$. Participants had no history of knee, thigh, hip or

110 lower back injuries within the past year and had not previously suffered an ACL tear. Participants provided

111 written informed consent to participate in the study, which was approved by the local ethics committee in-

112 line with the Helsinki Declarations for research with human volunteers. Prior to data collection, the

113 reproducibility of the featured assessments over three separate trials spaced by a 48-h period, was

114 established using 10 female university netball players who were part of the study cohort. The data was

115 analysed using procedures published by Hopkins ${ }^{19}$ to establish typical error as a percentage (TE \%). Mean

116 typical error as a percentage observed for each assessment is provided below.

\section{Experimental assessment protocol}

119 Before the assessments were conducted, all participants took part in a standardised warm-up consisting of 120 full-body static stretching followed by 5-min of jogging. All participants performed pre-testing; 20-m sprint $121(\mathrm{TE}=1.3 \%)$ recorded with Smart Speed electronic timing gates (Fusion Sport, Queensland, Australia). This 122 involved three maximal 20-m sprint attempts with 60-s rest in-between each sprint. Each sprint begin from 123 a standing start with the toe of the leading leg was placed behind start line, participants were instructed to 124 sprint as fast as possible on each sprint. Three individual countermovement jumps (CMJ) $(\mathrm{TE}=1.1 \%)$ with 125 60-s rest in-between. The maximal vertical displacement of the participant's jump was assessed with the 126 use of a Just Jump mat (Just Jump, Probotics, Huntsville, AL, USA). Participants were instructed to place 127 their hands on hips and when prompted by the assessor squat down to a self-selected depth, and jump 128 vertically as high as possible. The Illinois agility test $(\mathrm{TE}=2.7 \%)$ recorded with Smart Speed electronic 129 timing gates (Fusion Sport, Queensland, Australia), which involved three maximal test attempts with 180- 
130 s rest in-between each attempt. The test began from a standing start with the toe of the leading leg placed

131 behind start line, participants were instructed to complete the test as quickly as possible. For 20-m sprint,

$132 \mathrm{CMJ}$ and Illinois agility the mean value of the three maximal assessment attempts was used for subsequent 133 data analysis.

134 The QASLS was performed on both the right-leg $(\mathrm{TE}=2.0 \%)$ and left-leg $(\mathrm{TE}=2.3 \%)$ to assess

135 the dynamic neuromuscular control of the lower limb. All assessments were conducted by the same member 136 of the experimental team, who was experienced in using the assessment with athletes in an applied setting.

137 The protocol from Herrington and Munro $^{9}$ was adopted, which requires the single-leg squat movement to 138 be recorded and reviewed, scoring the movement at different regions of the body (arm, trunk, pelvis, thigh, 139 knee and foot). The associated scoring scale runs from $0-10$, a higher score indicates poor dynamic 140 neuromuscular control. ${ }^{9}$ This required each participant to position themselves $5-\mathrm{m}$ away from the video 141 camera (Sony Handycam CX250). Participants' were then required to stand on one leg and squat down to 142 a depth at which their knee angle was between $45^{\circ}-60^{\circ}$, they were asked to perform this movement over a 143 5-s period. The participants were given the opportunity to familiarise themselves with the depth of single144 leg squat with the use of a goniometer and three trials on each leg with a timer in front of them so they 145 stayed within the 5-s period. Participants were then required to perform three recorded single-leg squats on 146 each leg, and trials were only accepted if the squat was within the desired depth via goniometer monitoring.

147 Once the scores for each leg were collected, the three scores for each leg were averaged and this gave the 148 overall result for the left and right-leg. Both groups then completed post-testing, featuring all the described 149 assessments, following the training intervention (described below). Testing was performed two days after 150 training or competition to allow for sufficient recovery. All tests were carried out on basketball court 151 surface.

\section{Neuromuscular training intervention}

154 After pre-testing the intervention group engaged in a modified FIFA $11+$ neuromuscular training 155 programme, twice weekly for eight weeks (Table 1). The original FIFA 11+ protocol included football 
specific drills, subsequently these were removed from the current protocol and replaced with alternative exercises specific to court-based sports to increase the opportunity for neuromuscular improvements specific to the sports involved. ${ }^{20}$ All participants began each exercise of the training protocol at level one, progressions for an individual were not considered until their participation of the third session. Each session took 35-min to complete. During the intervention period both groups continued with their habitual training regimes as delivered by their sport coaches.

\section{INSERT TABLE 1 HERE}

\section{Statistical analysis}

Results are presented as mean ( \pm standard deviation) unless stated. A 2x2 mixed ANOVA was performed using the IBM SPSS version 22 to determine any significant differences $(\mathrm{p}<0.05)$ between the two groups performance measurements (20-m sprint, CMJ, Illinois agility test and QASLS). Assumptions of sphericity were assessed using Mauchly's test of sphericity, post-hoc pairwise-comparisons were conducted using the Bonferroni correction. Within effect size for intervention and control groups pre to post change on assessed measures was calculated using Cohen's $d$, with interpretation of observed effect sizes are as follows; trivial $<0.2$, small 0.2-0.6, moderate 0.6-1.2, large 1.2-2.0, very large $>2.0 .{ }^{21}$

\section{Results}

There was a significant effect over time for CMJ height $(\mathrm{F}=9.998, \mathrm{p}=0.005)$ and a significant trial $\mathrm{x}$ group interaction $\left(\mathrm{F}_{1,9}=36.571, \mathrm{p}<0.001\right)$. At post-testing $\mathrm{CMJ}$ significantly differed between the intervention and control group (6.58-cm [95\% CI: $2.48-10.69-\mathrm{cm}], \mathrm{p}=0.003 ; d=1.51)$. The intervention group's CMJ height was significantly increased from pre to post-testing $(3.96-\mathrm{cm}[95 \%$ CI: $2.68-5.24-\mathrm{cm}], \mathrm{p}<0.001$; $d=0.99$ ), whilst the control group experienced no significant change in from pre to post-testing CMJ height $(-1.36-\mathrm{cm}[95 \%$ CI: $-2.52--0.37-\mathrm{cm}], \mathrm{p}=0.056 ; d=-0.27)$.

There were no between-group differences for $20-\mathrm{m}$ sprint $\left(\mathrm{F}_{1,9}=1.704, \mathrm{p}=0.208\right)$. A significant within-group effect for 20-m performance was evident for the intervention group as performance 
182 significantly improved following the training intervention $(-0.09-\mathrm{s}$ [95\% CI: $-0.003--0.18-\mathrm{s}$ ], $\mathrm{p}=0.043, d$

$183=-0.40)$. No significant within-group effect was evident for the control group (-0.02-s [95\% CI: -0.10 -

$1840.08-\mathrm{s}], \mathrm{p}=0.747, d=0.07)$. There was no effect over time for the Illinois agility test $(\mathrm{F}=0.915, \mathrm{p}=0.351)$,

185 and no between-group differences $\left(\mathrm{F}_{1,9}=2.299, \mathrm{p}=0.147\right)$. There were no significant changes for either

186 group pre-to-post (Intervention group; -0.22-s [95\% CI: $-0.47-0.04-\mathrm{s}], \mathrm{p}=0.097, d=-0.32)$, (Control

187 group; 0.05-s [95\% CI: $-0.21-0.30-\mathrm{s}], \mathrm{p}=0.697, d=0.04)$.

There was a significant effect over time for QASLS right-leg scores $(F=14.814, p=0.001)$ and

left-leg scores $(\mathrm{F}=38.400, \mathrm{p}<0.001)$. A significant trial $\mathrm{x}$ group interaction was present for QASLS right-

190

191

192

193

194

195

196

197

198

199

200

201

202

203

204

205

leg scores $\left(\mathrm{F}_{1,9}=13.88, \mathrm{p}=0.002\right)$ and left-leg scores $\left(\mathrm{F}_{1,9}=50.00, \mathrm{p}<0.001\right)$. At post-testing QASLS scores for both the right-leg (2.22 [95\% CI: $1.09-3.25], \mathrm{p}=0.001 ; d=-1.30)$ and left-leg $(3.25$ [95\% CI: $2.00-4.50], \mathrm{p}<0.001 ; d=-1.98)$ were significantly different between the two groups. The intervention group reduced their right-leg $(-1.84[95 \% \mathrm{CI}:-1.12--2.56], \mathrm{p}=0.001 ; d=1.41)$ and left-leg $(-2.74[-2.13$ - -3.35], $\mathrm{p}<0.001 ; d=2.36)$ QASLS scores significantly from pre to post, the control group scores were unchanged (Right-leg; -0.03 [95\% CI: -0.75 - 0.69], $\mathrm{p}=0.931 ; d=0.02$, Left-leg; 0.18 [95\% CI: $-0.43-$ $0.79], \mathrm{p}=0.545 ; d=0.11)$.

\section{INSERT TABLE 2 HERE}

\section{Discussion}

The results demonstrate the FIFA 11+ protocol significantly increased CMJ height and reduced QASLS scores when compared to the control group outcomes, indicating improved lower limb neuromuscular control and stability which represents an improvement in a screening measure of ACL injury risk. ${ }^{1}$ Significant within-group improvements for the intervention group's CMJ, QASLS and 20-m sprint times were evidenced. Previous research has demonstrated neuromuscular training programmes can improve performance indicators such as sprint time, lower body power, agility, and proprioception as well as reduce the risk of ACL injury in female soccer and basketball populations. ${ }^{15,20,22}$

At post-testing the intervention group significantly increased their CMJ in comparison to the 
control group. There are several potential reasons why the CMJ height increased significantly within the intervention group. The featured neuromuscular programme included exercises such as box jumps and

210 depth jumps which are designed to elicit increases in lower body power and stretch shortening cycle

211 function. ${ }^{23}$ In addition, exercises such as planks, Nordic curls, unilateral balancing and bounding runs also

212 featured. Previous studies have highlighted the positive relationship between increased neuromuscular 213 activity within the core, hip and leg muscles all of which contribute towards the efficiency of a CMJ. ${ }^{12,13}$

214 Previous cohorts of athletes have recorded increased CMJ performance following prolonged interventions 215 utilizing the FIFA $11+$ programme. Bonato et $\mathrm{al}^{20}$ speculated an increase in postural control due to 216 performing the neuromuscular training programme may have attributed toward a significant increase in 217 CMJ height within female basketball athletes. Furthermore youth soccer populations performing FIFA 11+ intervention have experienced significant increases in CMJ height in comparison to control following interventions lasting 8-10 weeks. ${ }^{12,24}$

Following the neuromuscular programme there was a significant improvement in neuromuscular control observed during the QASLS test for both right and left-leg. These findings correspond with previous research regarding the benefit of neuromuscular training programmes for improving neuromuscular control and stability and reducing indictors of ACL injury risk in females. Bonato et $\mathrm{al}^{20}$ previously showed that a season-long, neuromuscular-training programme significantly improved postural control and reduced injury occurrence within female basketball players. While Benis et $\mathrm{al}^{13}$ found that twice weekly for neuromuscular training for 8 weeks, led to significant improvements in balance when compared to a control 227 group within female basketball players. In addition, researchers have reported a lower injury occurrence 228 within female youth soccer players following a season long, neuromuscular training programme. ${ }^{15,22}$ The 229 training protocol used within this study included exercises designed to elicit neural adaptations between the 230 motor cortex and the targeted muscle. ${ }^{11,12,13}$ The revised FIFA 11+ protocol was designed to condition the 231 following muscles: piriformis; superior and inferior gemelli; obturator internus / externus; quadratus 232 femoris; and the hamstrings and quadriceps muscle groups. Female athletes have been shown to have lower 233 recruitment of the muscles targeted by the intervention programme, which in turn cause misalignments in 
234 the lower body. ${ }^{3,10}$ Subsequently this places a significant amount of pressure on the ACL and its ability to 235 prevent anterior tibial subluxation, a common cause of ACL rupture. ${ }^{4,7}$ This study included exercises 236 specially chosen by Santa Monica Sports Medicine Foundation and the Oslo Trauma and Research Centre 237 (i.e. Nordic curls, unilateral balancing, box jumps, inline lunges and depth jumps) with the intention of 238 recruiting the aforementioned muscle groups responsible for protecting the integrity of the knee. ${ }^{14}$

A significant within-group reduction in 20-m sprint time pre to post for the intervention group was 240 apparent (2.4\% decrease). Similar to this study previous researchers have found the FIFA 11+ protocol or 241 similar training programmes to be effective for improving sprint performance; Kilding et $\mathrm{al}^{25}$ recorded a $2422 \%$ significant decrease in male soccer players $20-\mathrm{m}$ sprint time after performing the FIFA $11+$ training 243 programme five-times weekly for six weeks. In addition, Reis et al ${ }^{16}$ found significant improvements in $30-$ $244 \mathrm{~m}(3.3 \%$ reduction $)$ sprint for an intervention group of male futsal players performing the FIFA 11+ training 245 programme twice-weekly for 12 weeks. The intervention protocol within the present study included a combination of lower body strengthening exercises (inline lunges, Nordic curls) and plyometric exercises

247 (box jumps, depth jumps). Previous literature has found such exercises to invoke substantial muscular 248 recruitment and activation within hamstring, quadriceps and gluteal group musculature..$^{26,27,28,29}$ These such 249 muscle groups are strongly recruited during sprinting. ${ }^{30}$ Subsequently the observed enhancements in sprint 250 performance may have been due to an enhanced recruitment and firing rate of muscle fibres needed to 251 improve sprint mechanics and power output. ${ }^{11,12,13}$ However shorter (4 week) FIFA 11+ training 252 interventions have not shown such improvements in 20-m sprint time. ${ }^{31}$ Taking this study and other pieces 253 of evidence into consideration the FIFA $11+$ or a slightly amended version of the programme can be 254 considered a useful tool to maintain or reduce 20 -m sprint times in court-sport athletes. ${ }^{13,15,20}$ No significant differences between the intervention and control groups Illinois agility test time post eight weeks were discovered. The findings are in accordance with Kilding et $\mathrm{a}^{25}$ who found no significant 257 differences in Illinois agility test time following a FIFA 11+ training programme of 6 weeks duration when 258 compared to a control group within male soccer players. Since court-based athletes frequently engage in 259 change of direction cutting actions within their sport, they may have been less responsive to positively adapt 
to such movements within the training intervention. ${ }^{1}$ It is also important to note, a meta-analysis conducted by Gomes Neto et $\mathrm{al}^{18}$ who analyzed eleven FIFA $11+$ studies, including 4700 participants. The analysis 262 indicated an overall significant improvement in agility performance following engagement in the FIFA 11+ 263 programme. ${ }^{18}$ This may be indicative of the benefit of longitudinal implementation of the FIFA $11+$ on 264 agility.

The high adherence rate of participants within this study likely had a meaningful influence on the improvement in numerous post-intervention measures. In total $90 \%$ of the intervention group participants completed all 16 sessions. Participant adherence for neuromuscular training programmes which are relatively short in duration such as the one implemented within this study (approximately 35-min to complete), is essential for achieving improvements in physical performance and reducing the risk of sport related injuries. A systematic review supported this claim and concluded that neuromuscular training programmes approximately 25 -min duration with compliance rates of $75 \%$ significantly reduced the risk of

272 lower extremity injuries. ${ }^{32}$ Another study created three different groups to assess how adherence rates of the FIFA $11+$ programme affect the risk of injury. ${ }^{33}$ Participants where either assigned to unsupervised group (control), a group with a coach and physiotherapist (comprehensive) or a group with a coach and no physiotherapist (regular). The comprehensive, regular and control group achieved $86 \%, 81 \%$ and $73 \%$ completion of total possible sessions, respectively. The authors discovered that high player adherence to the intervention resulted in significant improvements in functional balance and a reduced injury risk. ${ }^{33}$ In conclusion, the results demonstrate a modified FIFA 11+ protocol can improve dynamic lower limb neuromuscular control and stability which represents an improvement in a screening measure of ACL injury risk. Considering that females are at greater risk of ACL injury in comparison to males and that the movements performed by court-sport athletes during performance can contribute further to the risk of an 282 ACL injury, implementation of preventative regimes such as the FIFA 11+ programme are justified. The 283 neuromuscular training programme also proved to significantly increase CMJ height in comparison to the 284 control group and lead to a within-group improvement in 20-m sprint for the intervention group. This 285 demonstrated the FIFA 11+ training programme can be used for improving desirable physical attributes for 
court-sport athletes

\section{Perspectives}

289 An amended FIFA 11+ programme performed twice weekly can be considered an appropriate 290 neuromuscular training regime to induce improvements in certain sport-specific performance measures and 291 neuromuscular control within a single-leg squat. This is in support of previous neuromuscular training 292 interventions which have shown to improve physical performance, proprioception and reduce the risk of 293 ACL injury in female soccer and basketball populations. ${ }^{15,20,22}$ Strength and conditioning practitioners 294 should explore the use of neuromuscular training programmes in court-based sports which have a high 295 prevalence of ACL injury especially in female populations. ${ }^{1,2,3,8}$ The QASLS scoring scale is considered a cost and time effective indirect screening method of assessing ACL injury risk, making it a practical and realistic tool for strength and conditioning practitioners. ${ }^{1,8,9}$ Considering that neuromuscular training programmes, such as the FIFA 11+ routine, require minimal equipment to conduct, they can be performed effectively as a strength and conditioning session and do not have to be performed as a warm-up routine alone.

\section{References}

1. Fox AS, Bonacci J, Saunders N. The relationship between performance of a single-leg squat and leap landing task: moving towards a netball-specific anterior cruciate ligament (ACL) injury risk screening method. Sports Biomech 2018;28:1-17.

2. James LP, Kelly VG, Beckman EM. Injury risk management plan for volleyball athletes: Sports Med 2014;44:1185-1195.

3. Hewett TE, Ford KR, Hoogenboom BJ, Myer GD. Understanding and preventing ACL injuries: current biomechanical and epidemiologic considerations-update 2010. N Am J Sports Phys 
Ther 2010;5:234-251.

4. Hirschmann M, Müller W, Hirschmann MT, Müller W. Complex function of the knee joint: the current understanding of the knee. Knee Surg Sports Traumatol Arthrosc 2015;23:27802788.

5. Elliot DL, Goldberg L, Kuehl KS. Young women's anterior cruciate ligament injuries: an expanded model and prevention paradigm. Sports Med 2010;40:367-376.

6. Vauhnik R, Morrissey MC, Rutherford OM, Turk Z, Pilih IA, Perme MP. Rate and risk of anterior cruciate ligament injury among sportswomen in Slovenia. J Athl Train 2011;46:92-98.

7. Shultz SJ. ACL Injury Risk in the Physically Active: Why are Females More Susceptible? Kinesiol Rev 2015;4:52-62. $\mathrm{K}$. Association between double-leg squat and single-leg squat performance and injury incidence among incoming NCAA Division I athletes: A prospective cohort study. Phys Ther Sport 2018;34:192-200. 2014;1:1-6.

9. Herrington L, Munro A. A preliminary investigation to establish the criterion validity of a qualitative scoring system of limb alignment during single-leg squat and landing. JESO

10. Hughes G, Dally N. Gender difference in lower limb muscle activity during landing and rapid change of direction. Sci Sport 2015;30:163-168. 
11. Zebis MK, Bencke J, Andersen LL, Døssing S, Alkjær T, Magnusson SP, Kjær M, Aagaard, P. The effects of neuromuscular training on knee joint motor control during side cutting in female elite soccer and handball players. Clin J Sport Med 2008;18:329-337.

12. Akbari H, Sahebozamani M, Daneshjoo A, Amiri-Khorasani M. Effect of the FIFA 11+ programme on vertical jump performance in elite male youth soccer players. MJSSM 2018;7:17-22.

13. Benis R, Bonato M, La Torre A. Elite Female Basketball Players' Body-Weight Neuromuscular Training and Performance on the Y-Balance Test. J Athl Train 2016;51:688-695. Mackler L, Dvorak J. Efficacy of the FIFA 11+ injury prevention program in the collegiate male soccer player. Am J Sports Med 2015;43:2628-2637. $\mathrm{R}$, Andersen TE. Comprehensive warm-up programme to prevent injuries in young female footballers: cluster randomised controlled trial. Br Med J 2008;337:a2469. Internationale de Football Association's "The 11+" injury prevention training program in youth futsal players. Clin J Sport Med 2013;23:318-320. Physiological and performance responses to the "FIFA 11+"(part 1): is it an appropriate warm- 
up? J Sports Sci 2013;31:1481-1490.

18. Gomes Neto M, Conceição CS, de Lima Brasileiro AJA, de Sousa CS, Carvalho VO, de Jesus FLA. Effects of the FIFA 11 training program on injury prevention and performance in football players: a systematic review and meta-analysis. Clin Rehabil 2017;31:651-659.

19. Hopkins WG. Analysis of reliability with a spreadsheet. [Online]. 2007; Available at: http://sportsci.org/resource/stats/ xrely.xls.

20. Bonato M, Benis R, La Torre A. Neuromuscular training reduces lower limb injuries in elite female basketball players. A cluster randomized controlled trial. Scand J Med Sci Sports 2018;28:1451-1460.

21. Hopkins WG. A scale of magnitudes for effect statistics. In: A New View of Statistics 2002; newstats.org/effectmag.html

22. Steffen K, Myklebust G, Olsen OE, Holme I, Bahr R. Preventing injuries in female youth football-a cluster-randomized controlled trial. Scand J Med Sci Sports 2008;18:605-614. Loturco I. Effects of plyometric training on neuromuscular performance in youth basketball players: a pilot study on the influence of drill randomization. J Sport Sci Med 2018;17:372378.

24. Rossler R, Donath L, Bizzini M, Faude O. A new injury prevention programme for children's football-FIFA 11+ Kids-can improve motor performance: a cluster-randomised controlled 
trial. J Sports Sci 2016;34:549-556.

391

25. Kilding AE, Tunstall H, Kuzmic D. Suitability of FIFA's “The 11" training programme for young football players-impact on physical performance. J Sport Sci Med 2008;7:320-326.

26. Asadi A. The effects of a 6-week of plyometric training on electromyography changes and performance. Sport Sci 2011;4:38-42.

27. Boudreau SN, Dwyer MK, Mattacola CG, Lattermann C, Uhl TL, McKeon JM. Hip-muscle activation during the lunge, single-leg squat, and step-up-and-over exercises. J Sport Rehabil 2009;18:91-103.

28. Ditroilo M, De Vito G, Delahunt E. Kinematic and electromyographic analysis of the Nordic Hamstring Exercise. J Electromyogr Kinesiol 2013;23:1111-1118.

29. Pincivero DM, Aldworth C, Dickerson T, Petry C, Shultz T. Quadriceps-hamstring EMG activity during functional, closed kinetic chain exercise to fatigue. Eur J Appl Physiol 2000;81:504-509.

30. Howard RM, Conway R, Harrison AJ. Muscle activity in sprinting: a review. Sports Biomech 2018;17:1-17.

31. Palazón FJR, Noguera CP, Rodríguez FA, Sánchez SH, Romero MTM, de Baranda MDPS, Wesolek I. Acute and chronic effects of the FIFA 11+ on several physical performance measures in adolescent football players. Eur J Hum Mov 2016;36:116-136. 
32. Ter Stege MH, Dallinga JM, Benjaminse A, Lemmink KA. Effect of interventions on potential, modifiable risk factors for knee injury in team ball sports: a systematic review. Sports Med 2014;44:1403-1426.

33. Steffen K, Emery CA, Romiti M, Kang J, Bizzini M, Dvorak J, Finch CF, Meeuwisse WH. High adherence to a neuromuscular injury prevention programme (FIFA 11+) improves functional balance and reduces injury risk in Canadian youth female football players: a cluster randomised trial. Br J Sports Med 2013;47:794-802. 


\section{Tables}

442 Table 1. Adapted version of FIFA 11+ protocol performed by the intervention group. Training session

443 exercises listed as sets $\mathrm{x}$ repetitions.

\begin{tabular}{|c|c|}
\hline Exercise and section & Sets and repetitions \\
\hline $\begin{array}{l}\quad \text { Warm-up (cones } 10 \text { m apart) } \\
\text { - } \text { Straight ahead } \\
\text { - } \text { Hip out } \\
\text { - } \text { Hip in } \\
\text { - } \text { Circle partner } \\
\text { - } \quad \text { Single-leg bounding } \\
\text { - } \quad \text { (added from main work out section) } \\
\text { - }\end{array}$ & $\begin{array}{l}\text { All exercises are to be performed } \\
\text { two times in the allocated area }\end{array}$ \\
\hline $\begin{array}{l}\text { Main training session } \\
\text { - } \quad \text { Plank (3 levels of difficulty) } \\
\text { - } \quad \text { Side plank (3 levels of difficulty) } \\
\text { - } \quad \text { Nordic curls (3 levels of difficulty) } \\
\text { - } \quad \text { Test your partners balance (single-leg) } \\
\text { (football removed) } \\
\text { - Box jumps (3 different heights) } \\
\text { - } \quad \text { Inline lunges (3 levels of difficulty) } \\
\text { - } \quad \text { Depth jumps (3 different heights) (added) } \\
\text { Running and cutting (3 levels of difficulty) }\end{array}$ & $\begin{array}{l}\text { 1 min rest between each set } \\
\text { - } 2 \times 60 \mathrm{~s} \\
\text { - } 2 \times 60 \mathrm{~s} \text { (each side) } \\
\text { - } 3 \times 6,8,10 \\
\text { - } 3 \times 60 \mathrm{~s} \text { (each leg) } \\
\text { - } 2 \times 30 \mathrm{~s} \\
\text { - } 2 \times 16 \text { (each leg) } \\
\text { - } 2 \times 6 \\
\text { - } 2 \text { sets within } 10 \mathrm{~m} \text { space }\end{array}$ \\
\hline Cool-down (added) & Full body static-stretch \\
\hline
\end{tabular}

444 
449 Table 2. Mean and (standard deviation) of all assessments pre and post-testing for both groups

\begin{tabular}{|c|c|c|c|c|}
\hline \multirow{2}{*}{ Assessment } & \multicolumn{2}{|c|}{ Control group } & \multicolumn{2}{|c|}{ Intervention group } \\
\hline & Pre & Post & Pre & Post \\
\hline 20-m (s) & $3.86(0.24)$ & $3.84(0.30)$ & $3.69(0.27)$ & $3.60(0.17)^{*}$ \\
\hline Illinois Agility (s) & $18.64(1.24)$ & $18.69(1.51)$ & $17.67(0.70)$ & $17.45(0.67)$ \\
\hline $\mathrm{CMJ}(\mathrm{cm})$ & $38.58(4.69)$ & $37.34(4.55)$ & $39.97(3.84)$ & $43.93(4.18)^{* \#}$ \\
\hline QASLS RL (1-10 scale) & $4.11(1.21)$ & $4.08(1.24)$ & $3.75(1.51)$ & $1.91(1.05)^{* \#}$ \\
\hline QASLS LL (1-10 scale) & $4.05(1.54)$ & $4.23(1.62)$ & $3.72(1.34)$ & $0.98(0.94)^{* \#}$ \\
\hline
\end{tabular}

450

$451 \quad{ }^{\#}=$ Significant interaction (group $x$ time) pre to post. $*$ = Significant within-group difference pre to post. 